\title{
441 POPULATION PHARMACOKINETICS OF AN ANTI-PD-1 ANTIBODY, PENPULIMAB IN PATIENTS WITH ADVANCED MALIGNANCIES
}

Benchao Chen, Xiaoping Jin, Yongcheng Dong, Max Wang, Dennis Xia, Michelle Xia, Baiyong Li*. Akeso Biopharma Co., Ltd., zhongshan, China

Background Penpulimab is a humanized IgG1 monoclonal antibody targeting PD-1 and is being evaluated in a variety of malignancies. The objective of the present study was to develop a population pharmacokinetic (PopPK) model of penpulimab in patients with hematologic malignancies and solid tumors, and to characterize the impact of demographic and disease factors on the pharmacokinetics.

Methods Pharmacokinetic data in patients with advanced malignancies were analyzed from six clinical studies with doses of $1,3,10 \mathrm{mg} / \mathrm{kg}, 200 \mathrm{mg}$ every 2 weeks (Q2W), or $200 \mathrm{mg}$ every 3 weeks $(\mathrm{Q} 3 \mathrm{~W})$ of penpulimab $(\mathrm{N}=332)$. Noncompartmental analyses were performed on PK data from a subset of participants and the pharmacokinetic characteristics of penpulimab were summarized.

Results The PopPK of penpulimab was adequately described with a two-compartment model with first-order elimination using a non-linear mixed effects model (NONMEM). Typical values for these $\mathrm{PK}$ parameters were estimated at $0.232 \mathrm{~L} /$ day for Clearance (CL), $3.42 \mathrm{~L}$ for the volume of distribution of central compartment (V1). There was no clinical correlation between penpulimab pharmacokinetics and baseline covariates, such as age, race, immunogenicity, hepatic function, creatinine clearance, tumor type, and ECOG score. Several indicators, including the impact of albumin, lactic dehydrogenase, and gender on CL, and the impact of weight and gender on V1, were found to be statistically significant, but the geometric mean ratios for the PK parameters ranged between 0.7 and 1.3 , with a difference of less than $30 \%$ and no clinically significant relevance. Drug exposure increased proportionally with dose from $1.0 \mathrm{mg} / \mathrm{kg}$ to $10.0 \mathrm{mg} / \mathrm{kg}$, and at $1 \mathrm{mg} / \mathrm{kg}$ or more, the PD-1 receptor occupancy reached $80 \%$ to $100 \%$ two days after penpulimab infusion. PopPK simulation showed that concentration ranges of penpulimab following administration at a fixed dose (200 mg Q2W) or by body weight (3.0 $\mathrm{mg} / \mathrm{kg}$ Q2W) were basically consistent. More than $97.5 \%$ of the patients, at either dosing regimen $(200 \mathrm{mg}$ Q2W or Q3W), had steady-state trough concentrations $>10 \mu \mathrm{g} / \mathrm{mL}$, which is much higher than the concentration $(0.5 \mu \mathrm{g} / \mathrm{mL})$ needed for reaching $90 \%$ to $100 \%$ PD-1 receptor occupancy.

Conclusions The PopPK model adequately assessed the pharmacokinetic characteristics and impact factors of penpulimab in the hematologic malignancies as well as solid tumors. This simulation indicates that both weight-based dose and fixed dose (200mg Q2W or Q3W) are appropriate for penpulimab. Ethics Approval These studies were conducted in accordance with the protocol, good clinical practice standards and the Declaration of Helsinki. The protocols and subsequent amendments were approved by the appropriate institutional review board (IRB) or ethics committee at each participating institution. All patients provided voluntary written informed consent. Consent Written informed consent was obtained from the patient for publication of this abstract and any accompanying images. A copy of the written consent is available for review by the Editor of this journal.

http://dx.doi.org/10.1136/jitc-2021-SITC2021.441 